\title{
Bombesin-functionalized superparamagnetic iron oxide nanoparticles for dual-modality MR/NIRFI in mouse models of breast cancer
}

This article was published in the following Dove Press journal: International Journal of Nanomedicine

\author{
Li Li ${ }^{1, *}$ \\ Changqiang $\mathrm{Wu}^{2, *}$ \\ Lili Pan' \\ Xin $\mathrm{Li}^{1}$ \\ Anren Kuang' \\ Huawei Cai \\ Rong Tian' \\ 'Department of Nuclear Medicine, West \\ China Hospital, Sichuan University, \\ Chengdu 6I004I, People's Republic of \\ China; ${ }^{2}$ Sichuan Key Laboratory of \\ Medical Imaging \& School of Medical \\ Imaging, North Sichuan Medical College, \\ Nanchong 637000, People's Republic of \\ China
}

*These authors contributed equally to this work
Correspondence: Huawei Cai

Department of Nuclear Medicine, West

China Hospital, Sichuan University, No. 37,

Guoxue Lane, Wuhou District, Chengdu

6I004I, People's Republic of China

Tel +86 I $38807 \mid 1669$

Email hw.cai@yahoo.com

Rong Tian

Department of Nuclear Medicine, West China Hospital, Sichuan University, No. 37, Guoxue Lane, Wuhou District, Chengdu 61004I, People's Republic of China

Tel +86 I 898060 I586

Fax +86 02885422187

Email rongtiannuclear@126.com
Background: The early and accurate detection afforded by imaging techniques significantly reduces mortality in cancer patients. However, it is still a great challenge to achieve satisfactory performance in tumor diagnosis using any single-modality imaging method. Magnetic resonance imaging (MRI) has excellent soft tissue contrast and high spatial resolution, but it suffers from low sensitivity. Fluorescence imaging has high sensitivity, but it is limited by penetration depth. Thus, the combination of the two modes could result in synergistic benefits. Here, we design and characterize a novel dual-modality MR/nearinfrared fluorescence imaging (MR/NIRFI) nanomicelle and test its imaging properties in mouse models of breast cancer.

Methods: The nanomicelles were prepared by incorporating superparamagnetic iron oxide (SPIO) nanoparticles into 1,2-distearoyl-sn-glycero-3-phosphoethanolamine-N-[amino(polyethylene glycol)-5000] micelles to which an NIRF dye and a tumor-targeted peptide $\left(\mathrm{N}_{3}\right.$-Lysbombesin, Bom) were conjugated. The nanomicelles were characterized for particle size, zeta potential and morphology. The transverse relaxivity, targeting specificity and imaging ability of the nanomicelles for MR/NIRFI were also examined.

Results: The fabricated nanomicelles displayed a well-defined spherical morphology with a mean diameter of $145 \pm 56 \mathrm{~nm}$ and a high transverse relaxivity $\left(493.9 \mathrm{mM}^{-1} \cdot \mathrm{s}^{-1}, 3.0 \mathrm{~T}\right)$. In MRI, the $T_{2}$ signal reduction of tumors in the Bom-targeted group was $24.1 \pm 5.7 \%$ at $4 \mathrm{hrs}$ postinjection, whereas only a $0.1 \pm 3.4 \%(P=0.003)$ decrease was observed in the nontargeted group. In NIRFI, the contrast increased gradually in the targeted group, and the tumor/muscle ratio increased from $3.7 \pm 0.3$ at $1 \mathrm{hr}$ to $4.7 \pm 0.1$ at $2 \mathrm{hrs}$ and to $6.4 \pm 0.2$ at $4 \mathrm{hrs}$. No significant changes were observed in the nontargeted group at any time points.

Conclusion: Considering all our results, we conclude that these novel MR/NIRFI dualmodality nanomicelles could be promising contrast agents for cancer diagnosis.

Keywords: SPIO nanoparticles, magnetic resonance imaging, near-infrared fluorescence imaging, tumor diagnosis

\section{Introduction}

Cancer remains a great challenge to global public health and a tremendous economic burden on society. In China, cancer is the leading cause of death, with an estimated 4.3 million new cases and 2.9 million deaths in 2015 . $^{1}$ According to the World Cancer Report 2014, between one-third and one-half of cancer deaths could be avoided with prevention, early detection and treatment. ${ }^{2}$ Noninvasive imaging methods, including magnetic resonance imaging (MRI), computed tomography, optical imaging and positron emission tomography, play an important role in 
early detection. ${ }^{3,4}$ Among them, MRI is one of the most commonly used techniques. It provides the advantages of multiparameter imaging, excellent soft tissue contrast and high spatial resolution with no exposure to ionizing radiation. ${ }^{5,6}$ Although MRI has become a powerful technique in cancer diagnosis, it suffers from low sensitivity. Optical imaging, such as near-infrared fluorescence imaging (NIRFI), is low in cost, provides images with high sensitivity and enables real-time imaging, but exhibits poor spatial resolution. ${ }^{7,8}$ NIRFI detects fluorescence emissions in the region of $650-900 \mathrm{~nm}$, has low background tissue absorption for deeper penetration depth and is more suitable for in vivo preclinical and clinical imaging studies. ${ }^{7,9}$ Therefore, the development of an MR/ NIRFI dual-modality molecular imaging probe might be able to combine the advantages of both imaging technologies and enable better diagnostic performance for patients with cancer.

Superparamagnetic iron oxide (SPIO) nanoparticles, because of their low toxicity, inherent magnetic properties and feasibility to produce iron oxide cores, have been intensively investigated as promising MRI probes for $T_{2}$-weighted (dark or negative contrast) imaging. ${ }^{3,10-14}$ Adding near-infrared (NIR) fluorophores to SPIO agents can overcome the intrinsic drawbacks of both imaging techniques while achieving improved detection accuracy, and allows the easy and accurate evaluation of the biodistribution of SPIO agents, even at low levels. ${ }^{4,15}$ However, the discrepancy in sensitivity between the two imaging modalities proves to be a challenge to the design of dual-modality MR/NIRFI probes. ${ }^{16}$ Polymeric micelles can be used to alleviate this discrepancy by increasing the number of MRI contrast units loaded into the hydrophobic micelle core relative to the number of fluorophores. ${ }^{16,17}$ The resulting high loading density of the SPIO agents allows the detection of probes at nanomolar concentrations. ${ }^{17}$ Furthermore, when incorporated into micelles, SPIO nanoparticles achieve a longer blood halflife, better biocompatibility and improved surface modification. ${ }^{18-20}$ Among the polymeric micelles, 1,2-distearoyl-sn-glycero-3-phosphoethanolamine-poly(ethylene glycol) (DSPE-PEG) micelles achieve the highest transportability and have been approved by the FDA for medical applications. ${ }^{19,21}$

The surface of DSPE-PEG micelles can be easily conjugated with targeting moieties, promoting the specific accumulation of SPIO nanoparticles in tumor tissue. Targeting agents, including antibodies, proteins, peptides, aptamers and small organic molecules, have been used in
SPIO nanoparticle systems. ${ }^{16}$ The advantages of easy synthesis, relatively small molecular weight and low cytotoxicity and immunogenicity make peptides particularly attractive for the purpose of targeting. ${ }^{16,22}$ Among them, bombesin is a 14 amino-acid peptide isolated from frog skin and shares a homologous C-terminal seven aminoacid sequence with mammalian gastrin-releasing peptide and neuromedin B. Therefore, the bombesin receptor family in mammals is comprised of gastrin-releasing peptide receptor (GRPR), neuromedin B receptor and bombesin receptor subtype $3 .^{23}$ These bombesin receptors, particularly GRPR, have been shown to be one of the most overexpressed or ectopically expressed families of $G$ protein-coupled receptors in many common malignancies, including breast cancer, lung cancer, prostate cancer, pancreatic cancer, head/neck cancer, colon cancer, uterine cancer, ovarian cancer, renal cell cancer, glioblastoma, neuroblastoma, gastrointestinal carcinoids and bronchial carcinoids. ${ }^{24,25}$ Many of these malignancies have a poor prognosis with advanced disease, and thus, there is heightened interest in the possibility of using GRPR overexpression not only to localize the tumor but also to deliver cytotoxic agents. ${ }^{24-26}$ Prior to this work, there were only four previous reports of bombesin-functionalized SPIO nanoparticles with dextran or chitosan coatings for targeting prostate cancer, breast cancer or normal pancreatic cells in vitro and/or in vivo. ${ }^{27-30}$

In this study, using SPIO nanoparticles as an MRI contrast agent, 1,2-distearoyl-sn-glycero-3-phosphoethanolamine-N-[amino(polyethylene glycol)-5000] (DSPE-PEG5k) micelles as a nanocarrier, $\mathrm{Cy} 5$ as an NIR fluorophore and $\mathrm{N}_{3}$ Lys-bombesin (Bom) with a sequence of $\left\{\operatorname{Lys}\left(\mathrm{N}_{3}\right)\right\}$ QRLGNQWAVGHLM as a targeting peptide, a dual-modality MR/NIRFI nanoprobe SPIO/DSPE-PEG5k-(Bom\&Cy5) was constructed for tumor diagnosis. The size, zeta potential, morphology and transverse relaxivity of the SPIO/DSPEPEG5k-(Bom\&Cy5) nanomicelles were investigated. The toxicity and targeting efficiency in vitro and in vivo were evaluated. Moreover, the dual-modality imaging properties of the nanomicelles in vivo were tested using MDA-MB-231 breast xenograft models in nude mice.

\section{Materials and methods}

\section{Materials}

Hydrophobic SPIO nanoparticles were synthesized by our laboratory and stored in hexane according to a previously reported method. ${ }^{31}$ DSPE-PEG5k was purchased from 
Avanti Polar Lipids, Inc. (Alabaster, AL, USA). The NIR dye Cy5-N-hydroxysuccinimide (Cy5-NHS) was obtained from Xi'an Ruixi Biological Technology Co., Ltd. (Xi'an, Shaanxi, China). The peptide Bom with a sequence of $\left\{\operatorname{Lys}\left(\mathrm{N}_{3}\right)\right\}$ QRLGNQWAVGHLM was synthesized by GenScript Biotech Corp. (Piscataway, NJ, USA). Cell Counting Kit-8 (CCK-8) was purchased from Dojindo Molecular Technologies, Inc. (Shanghai, China). All reagents were of analytical grade and were used as received.

\section{General procedure for the synthesis of SPIO/DSPE-PEG5k-(Bom\&Cy5)}

DSPE-PEG5k (10 mg, $1.7 \mu \mathrm{mol})$ was dissolved in $10 \mathrm{~mL}$ of dry tetrahydrofuran (THF). Propargyl bromide (2 mg, $17 \mu \mathrm{mol})$ and triethylamine $(2.5 \mathrm{mg}, 26 \mu \mathrm{mol})$ were added, and the solution was stirred and heated to $50^{\circ} \mathrm{C}$ for $24 \mathrm{hrs}$. After the solvents were evaporated, the residue was dissolved in $10 \mathrm{~mL}$ of dichloromethane and washed three times with water. The organic phase was dried over sodium sulfate and removed to obtain alkynyl-modified DSPE-PEG5k (DSPE-PEG5k-Alky). Then, $4.5 \mathrm{mg}$ of hydrophobic SPIO nanoparticles, $3 \mathrm{mg}$ of DSPE-PEG5k and $3 \mathrm{mg}$ of DSPE-PEG5k-Alky were dissolved in $1 \mathrm{~mL}$ of THF, and the mixture was added to $10 \mathrm{~mL}$ of Milli-Q water under probe sonication. SPIO/DSPE-PEG5k nanomicelles in water $(10 \mathrm{~mL})$ were obtained after the evaporation of THF.

Bom (1.4 mg) was dissolved in $0.2 \mathrm{~mL}$ of Milli-Q water and mixed with the obtained SPIO/DSPE-PEG5k solution $(5 \mathrm{~mL}) . \mathrm{CuCl}_{2}(1.5 \mathrm{mg})$ and sodium ascorbate $(19 \mathrm{mg}$ ) were then added, and the reaction mixture was heated in an oil bath at $50^{\circ} \mathrm{C}$ for 1 day. Then, $1 \mathrm{mg}$ of Cy5NHS (dissolved in $1 \mathrm{~mL}$ DMSO) was added and stirred at room temperature for $24 \mathrm{hrs}$ in the dark. The expected SPIO/DSPE-PEG5k-(Bom\&Cy5) was collected after the resulting solution was dialyzed for $24 \mathrm{hrs}$ against water (MWCO $10 \mathrm{kDa}$ cutoff).

\section{Characterization of the nanomicelles}

The morphology and structure of the nanomicelles were characterized by transmission electron microscopy (TEM) (H-7500; Hitachi, Tokyo, Japan). The size distribution and zeta potential were characterized by dynamic light scattering (DLS) using a Malvern Zetasizer (Malvern Instruments, Malvern, UK) at $25^{\circ} \mathrm{C}$. The peptide content was determined using a bicinchoninic acid (BCA) assay, and the iron concentration was determined by furnace atomic absorption spectroscopy (AA800; PerkineElmer, Akron, OH, USA).

\section{Relaxivity and magnetization measurement}

The MR relaxation data were acquired using a 3.0 Tesla MR scanner (Achieva; Philips, Amsterdam, Netherlands). Solutions of SPIO/DSPE-PEG5k-(Bom\&Cy5) were prepared at final Fe concentrations of $0,0.05,0.1,0.2,0.3$, 0.4 and $0.5 \mathrm{mM}$. The $T_{2}$-weighted images were acquired with a spin echo sequence $(\mathrm{TR}=5000 \mathrm{~ms}$; TE ranging from 12 to $500 \mathrm{~ms})$. Transverse relaxation times $\left(T_{2}\right)$ were calculated by fitting a logarithmic regression to the signal intensity (SI) in images at different echo times. The relaxivity value $\left(r_{2}\right)$ was determined from the slope of the transverse relaxation rate $\left(1 / T_{2}, \mathrm{~s}^{-1}\right)$ versus the Fe concentration (mM).

Magnetic studies were carried out using an MPMS7 Quantum Design SQUID magnetometer (Quantum Design, San Diego, CA, USA) at $300 \mathrm{~K}$. A sample of SPIO/DSPEPEG5k-(Bom\&Cy5) solution was lyophilized and then measured with the scope of -3 T to $3 \mathrm{~T}, 4$ quadrants.

\section{Cell culture}

The human breast cancer cell line MDA-MB-231 was purchased from the Cell Bank of the Chinese Academy of Science and cultured in Dulbecco's modified Eagle's medium (HyClone, Beijing, China) supplemented with 1\% penicillin/streptomycin (Gibco, Shanghai, China) and 10\% fetal bovine serum (Gibco, Shanghai, China). The cultured cells were maintained at $37^{\circ} \mathrm{C}$ and $5 \% \mathrm{CO}_{2}$ in a tissue culture incubator. The human vascular endothelial cell line HUVEC, human hepatic cell line L02 and human embryonic kidney cell line HEK-293 were obtained from the Key Laboratory of Transplant Engineering and Immunology of Sichuan University. These cell lines were cultured under identical conditions to those of the MDA-MB-231 cell line. Cell lines were routinely tested to exclude mycoplasma contamination and characterized by Chengdu Nuohe Biotech Co., Ltd (Chendu, Sichuan, China) and Shanghai Cinoasia Institute (Shanghai, China) using short tandem repeat markers.

\section{Targeting efficiency in vitro}

To evaluate the targeting capability of the SPIO/DSPEPEG5k-(Bom\&Cy5) nanomicelles in vitro, MDA-MB-231 cells were seeded in a $24-w e l l$ plate containing coverslips 
at a density of $3 \times 10^{4}$ cells per well. After incubation overnight, the solutions were replaced with serum-free medium containing Bom-targeted nanomicelles $(10 \mu \mathrm{g}$ $\mathrm{Fe} / \mathrm{mL})$ or nontargeted nanomicelles $(10 \mu \mathrm{g} \mathrm{Fe} / \mathrm{mL})$. For the blocking experiment, a tenfold excess of free Bom $(100 \mu \mathrm{g} / \mathrm{mL})$ was added to the culture media and incubated at $37^{\circ} \mathrm{C}$ for $2 \mathrm{hrs}$ prior to the addition of Bom-targeted nanomicelles. After incubation for $4 \mathrm{hrs}$, the cells were gently washed three times with PBS and then fixed with $4 \%$ paraformaldehyde for $10 \mathrm{~min}$. Then, the cells were washed with PBS again, and the nuclei were stained with DAPI $(10 \mu \mathrm{g} / \mathrm{mL})$ for 10 mins. After fixing and dye administration, the cells were washed three times with PBS and observed under a fluorescence microscope (Imager Z2; Zeiss, Oberkochen, Germany).

\section{In vitro cytotoxicity assay}

The HUVEC, L02 and HEK-293 cell lines, which are associated with the metabolism of nanoparticles in vivo, were employed to evaluate the cytotoxicity of the SPIO/ DSPE-PEG5k-(Bom\&Cy5) nanomicelles using the CCK-8 assay. Cells were seeded at a density of $5 \times 10^{3}$ cells per well in 96-well plates and incubated for $24 \mathrm{hrs}$. Then, the cells were treated with cell culture medium containing gradient concentrations of the nanomicelles (equivalent to $0,1,2,5,10,20$ and $40 \mu \mathrm{g} \mathrm{Fe} / \mathrm{mL} ; \mathrm{n}=5$ ) and incubated for $24 \mathrm{hrs}$. Untreated cells in growth media were used as a control. Then, $10 \mu \mathrm{L}$ of CCK-8 solution was added to each well, followed by incubation for $4 \mathrm{hrs}$. The absorbance intensity was measured at $450 \mathrm{~nm}$ using a microplate reader (Huadong Electronics, Nanjing, Jiangsu, China).

\section{In vivo toxicity analysis}

For the in vivo toxicity study, BALB/c mice were obtained from Beijing Vital River Laboratory Animal Technology Co., Ltd. (Beijing, China). All animal procedures were performed in accordance with the Guidelines for the Care and Use of Laboratory Animals of Sichuan University, and experiments were approved by the Animal Ethics Committee of West China Hospital of Sichuan University. Mice were separated into three groups $(\mathrm{n}=3)$. SPIO/DSPE-PEG5k-(Bom\&Cy5) at a total dose of $300 \mu \mathrm{g} \mathrm{Fe} / \mathrm{mL}(200 \mu \mathrm{L})$ was injected into $\mathrm{BALB} / \mathrm{c}$ mice via the tail vein. The untreated mice were used as the control. The mice were sacrificed, and the organs (heart, lung, liver, spleen and kidney) were harvested on the 3rd, 7th or 21 st day after the nanomicelles were delivered. The collected tissues were embedded in paraffin, sectioned (5 $\mu \mathrm{m}$ thick) and then stained with hematoxylin and eosin (H\&E) for histological analysis. The stained sections were observed under an optical microscope (Leitz Optilux; Leica, Wetzlar, Germany). Before the mice were euthanized, blood samples were drawn from the eye socket for blood biochemistry testing.

\section{In vivo targeting and imaging performance}

The breast tumor xenograft model was established by the subcutaneous injection of an MDA-MB-231 cell suspension $\left(1 \times 10^{6}\right.$ cells $\left./ 100 \mu \mathrm{L}\right)$ with $30 \%$ basement membrane matrix into the right axillary region of each female BALB/c nude mouse. MR and NIRFI studies were carried out when the tumors had grown to $5-10 \mathrm{~mm}$ in diameter ( 2 weeks after inoculation). Mice were fed an alfalfa-free diet (AIN-93G; OpenSource Diets, New Brunswick, NJ, USA) in order to reduce autofluorescence from the intestine. ${ }^{32}$ Two hundred microliters $(100 \mu \mathrm{g} \quad \mathrm{Fe} / \mathrm{mL})$ of SPIO/DSPE-PEG5k(Bom\&Cy5) or SPIO/DSPE-PEG5k-Cy5 solution was injected via the tail vein. All experiments were conducted under complete anesthesia using $3 \%$ isoflurane.

$T_{2}$-weighted MRI of the animals was performed preinjection and 1, 2 and $4 \mathrm{hrs}$ postinjection using a 3.0 Tesla clinical MRI system (Achieva; Philips, Amsterdam, Netherlands). $T_{2}$-weighted fast spin echo images were acquired with the following parameters: $\mathrm{TR}=4000 \mathrm{~ms}, \mathrm{TE}=66 \mathrm{~ms}, \mathrm{FOV}=50$ $\mathrm{mm} \times 50 \mathrm{~mm}$ and slice thickness $=1 \mathrm{~mm}$. The MRI contrast change in the tumor following the injection of the nanomicelles was quantitatively calculated using the region of interest (ROI) method with ImageJ 1.8.0. The normalized SI (NSI) was obtained by dividing the SI of the tumor ROI by the SI of the water phantom ROI. For NIRFI, nanomicelles were injected into the tail vein of the tumor-bearing mouse to obtain images at the same time points postinjection using a fluorescence imaging system (IVIS Spectrum; Caliper Life Sciences, Alameda, CA, USA). The fluorescent SI was analyzed using Living Image 4.4 software (Caliper Life Sciences, Alameda, CA, USA).

\section{Histological analysis}

The mice were euthanized following MR/NIRFI to collect the tumors for histological analysis. The tissues were fixed with $10 \%$ neutral buffered formalin, embedded in paraffin and then sectioned ( $5 \mu \mathrm{m}$ thick). Prussian blue staining was performed to detect iron accumulation in the tissue sections. Then, images were obtained by fluorescence microscopy (Imager Z2; Zeiss, Oberkochen, Germany). 


\section{Statistical analysis}

Data are presented as the mean \pm standard deviation and were analyzed using SPSS 22.0 software (IBM, Armonk, NY, USA). Student's $t$-test was utilized for statistical evaluation. A statistically significant difference was defined as a value of $P<0.05$.

\section{Results}

\section{Preparation and characterization of the nanomicelles}

A schematic diagram of the preparation of SPIO/DSPEPEG5k-(Bom\&Cy5) is illustrated in Figure 1. Briefly, the SPIO nanoparticles were synthesized through thermal decomposition, as reported by Sun et $\mathrm{al}^{31}$, and then functionalized with polymeric micelles self-assembled by DSPEPEG5k and DSPE-PEG5k-Alky. Bom and Cy5-NHS were then introduced into the reaction medium in different steps. The morphology, structure, size distribution and zeta potential were measured using TEM and DLS. ATEM image of the SPIO/DSPE-PEG5k-(Bom \&Cy5) nanomicelles is shown in Figure 2A. The image demonstrated the successful entrapment of the SPIO nanoparticles into the polymeric micelles, with the nanomicelles exhibiting a well-defined spherical morphology (Figure 2A). The average diameter of the nanomicelles without Bom was $131 \pm 45 \mathrm{~nm}$, and Bom conjugation increased the diameter to $145 \pm 56 \mathrm{~nm}$ (Figure 2B). In addition, the zeta potential with and without Bom were similar at $-2.9 \pm 1.1 \mathrm{mV}$ and $-0.2 \pm 0.8 \mathrm{mV}$, respectively. Quantification of the Bom concentration using a BCA assay showed that the amount of Bom conjugated to the nanomicelle surface was $94 \mu \mathrm{g}$ per $100 \mu \mathrm{g} \mathrm{Fe}$.

\section{Relaxivity and magnetization measurement}

The magnetic relaxivity is an important parameter of MRI contrast agents. The $r_{2}$ of the nanomicelles was examined using $T_{2}$-weighted MRI on a 3.0 Tesla MR scanner. The negative signal of the SPIO/DSPE-PEG5k(Bom\&Cy5) nanomicelle samples gradually increased as the $\mathrm{Fe}$ concentration increased from 0 to $0.5 \mathrm{mM}$ (Figure $3 \mathrm{~A}$ ). The $1 / T_{2}$ values of the nanomicelles demonstrated a linear dependency on the Fe concentration (Figure 3B), with a high $r_{2}$ of $493.9 \mathrm{mM}^{-1} \cdot \mathrm{s}^{-1}$, indicating that these nanomicelles can effectively serve as $T_{2}$ contrast agents.

The magnetic hysteresis of the SPIO nanoparticles and SPIO/DSPE-PEG5k-(Bom\&Cy5) nanomicelles were measured using the SQUID magnetometer. Figure 3C confirms
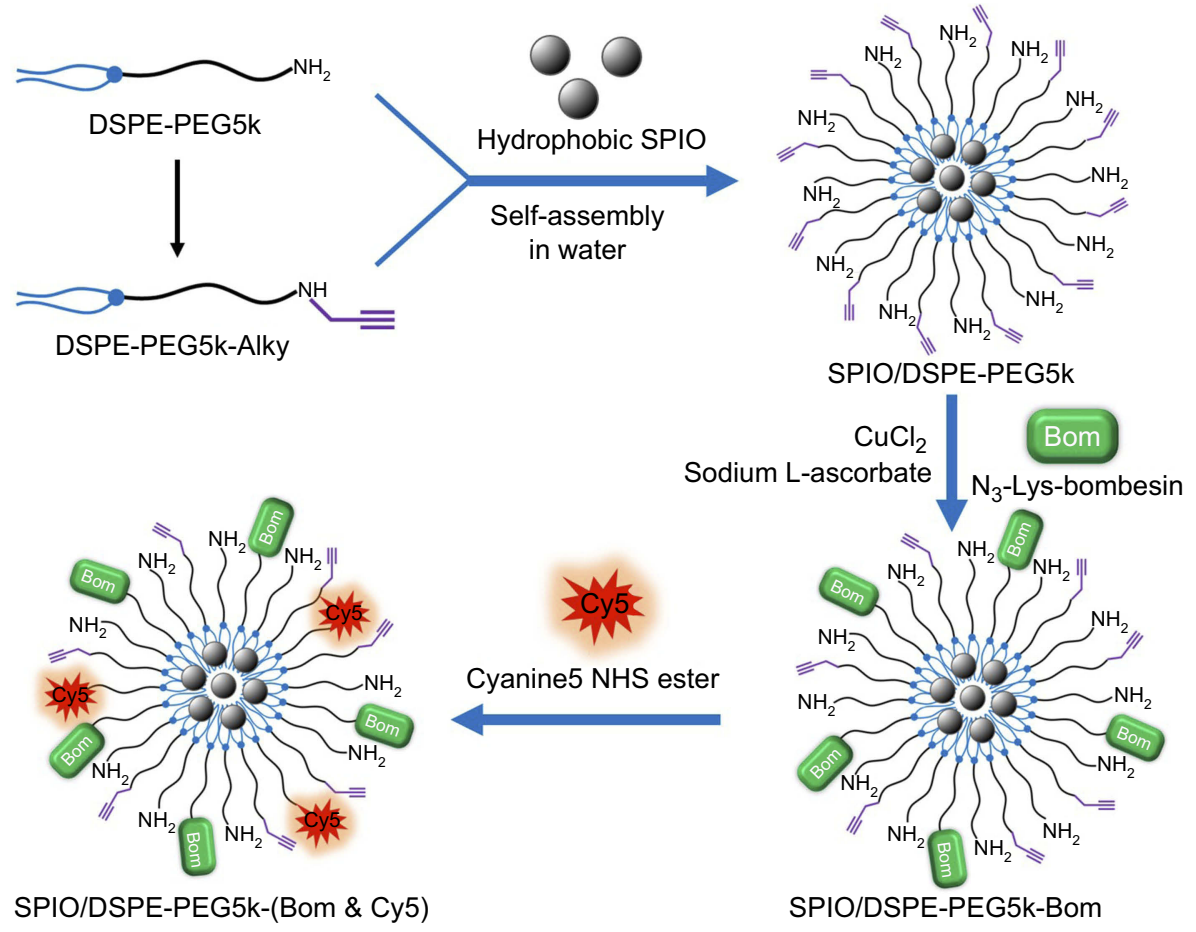

Figure I Schematic diagram for the synthesis of SPIO/DSPE-PEG5k-(Bom\&Cy5).

Abbreviations: SPIO, superparamagnetic iron oxide; NHS, N-hydroxysuccinimide. 

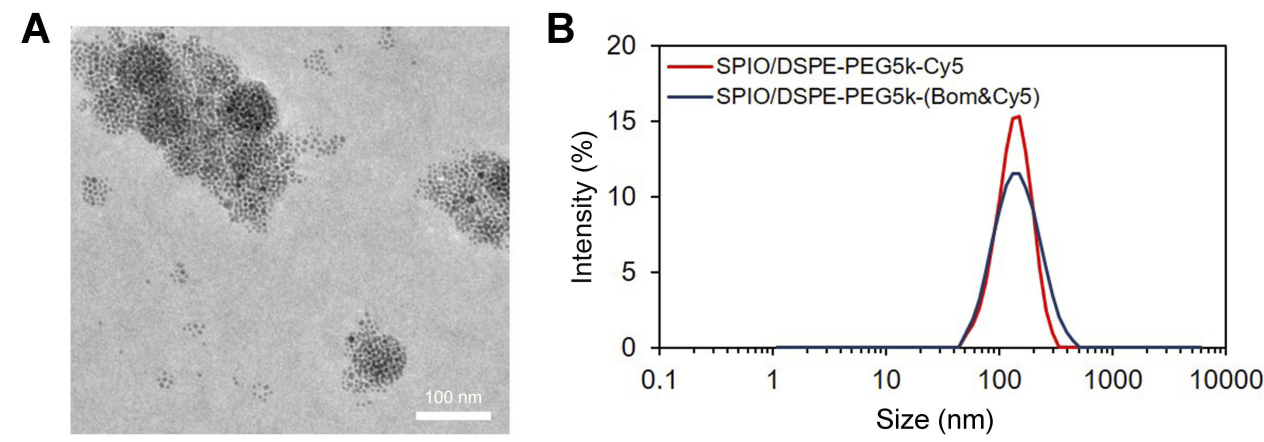

Figure 2 Nanomicelle characterization.

Notes: (A) A TEM image of Bom-targeted nanomicelles (scale bar $=100 \mathrm{~nm}$ ). (B) Size distribution of Bom-targeted (blue) and nontargeted (red) nanomicelles in aqueous solution measured by DLS.

Abbreviations: TEM, transmission electron microscopy; DLS, dynamic light scattering.
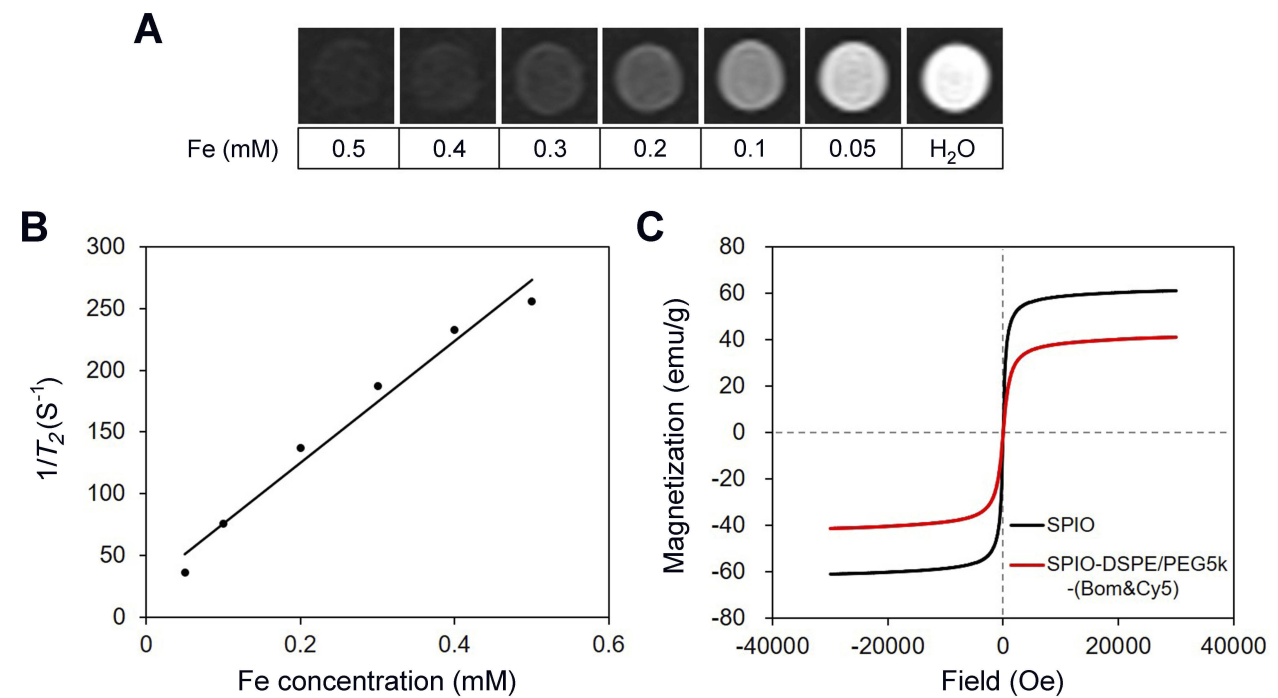

Figure 3 Relaxivity and magnetization measurement.

Notes: $\left(\right.$ A) $T_{2}$-weighted images of SPIO/DSPE-PEG5k-(Bom\&Cy5) nanomicelle samples at different iron concentrations (3.0 T, spin-echo sequence: TR =5000 ms, TE = 12 ms). (B) Chart of the change in $\mathrm{I} / T_{2}$ values with Fe concentration. (C) Hysteresis loops of the SPIO nanoparticles (black) and SPIO/DSPE-PEG5k-(Bom\&Cy5) nanomicelles (red) measured at $300 \mathrm{~K}$.

Abbreviation: SPIO, superparamagnetic iron oxide.

the superparamagnetic behavior of the two samples. Under a strong external magnetic field, the magnetization of the two samples aligns with the field direction and reaches its saturation value. The saturation magnetization values of the SPIO nanoparticles and SPIO/DSPE-PEG5k(Bom\&Cy5) nanomicelles were $61.0 \mathrm{emu} / \mathrm{g}$ and 41.2 $\mathrm{emu} / \mathrm{g}$, respectively. The net magnetization of the two samples returned to zero in the absence of an external field, indicating they are superparamagnetic at room temperature $(300 \mathrm{~K})$.

\section{Cytotoxicity and in vivo toxicology}

The HUVEC, L02 and HEK-293 cell lines, which are associated with the metabolism of nanoparticles in vivo, were chosen as model cells. The in vitro cytotoxicity of the SPIO/DSPE-PEG5k-(Bom\&Cy5) nanomicelles for these cell lines was investigated using the CCK- 8 assay. As shown in Figure 4A, the nanomicelles exhibited negligible cytotoxicity against HEK-293, HUVEC and L02 cells at various $\mathrm{Fe}$ concentrations ranging from 0 to 40 $\mu \mathrm{g} / \mathrm{mL}$ for $24 \mathrm{hrs}$, which indicated good biocompatibility of the nanomicelles. The potential long-term toxicity of nanomedicine is an essential concern for biomedical applications. Further experiments in vivo, including blood biochemistry analysis and histology examination, were conducted to evaluate the long-term toxicity of the SPIO/ DSPE-PEG5k-(Bom\&Cy5) nanomicelles. As displayed in Figure 4B, the serum parameters of treated mice, including 

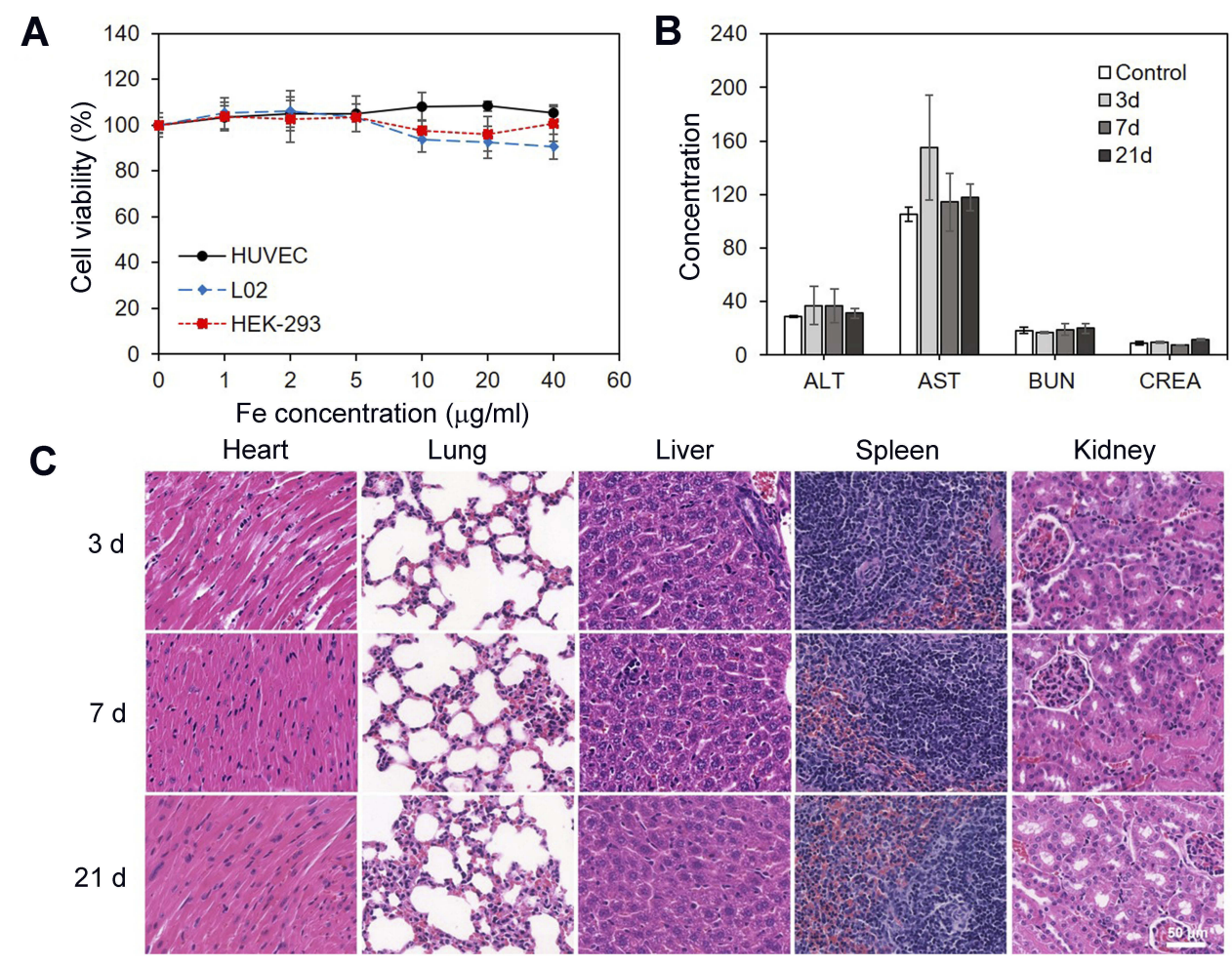

Figure 4 Cytotoxicity and in vivo toxicology.

Notes: (A) In vitro cell viability of HUVEC, L02 and HEK-293 cells after incubation with various concentrations of SPIO/DSPE-PEG5k-(Bom\&Cy5) nanomicelles for 24 hrs. (B) Serum biochemistry data on ALT, AST, BUN and CREA. (C) Micrographs of H\&E-stained organ slices (heart, lung, liver, spleen and kidney) from mice 3 days, 7 days and 2I days after intravenous injection of SPIO/DSPE-PEG5k-(Bom\&Cy5) nanomicelles (H\&E staining, 40x). Scale bar $=50 \mu \mathrm{m}$.

Abbreviations: SPIO, superparamagnetic iron oxide; ALT, alanine aminotransferase; AST, aspartate aminotransferase; BUN, blood urea nitrogen; CREA, creatinine.

liver function markers (alanine aminotransferase (ALT) and aspartate aminotransferase (AST)) and kidney function indicators (blood urea nitrogen (BUN) and creatinine (CREA)), were consistent with those of the control groups. In addition, no noticeable abnormalities or tissue damage in the different organs (heart, lung, liver, spleen and kidney) were detected after 3, 7 and 21 days (Figure 4C), indicating that the SPIO/DSPE-PEG5k-(Bom\&Cy5) nanomicelles exerted nonvisible effects in vivo. All the results suggest that the SPIO/DSPE-PEG5k-(Bom\&Cy5) nanomicelles are a high-performance contrast agent with negligible long-term toxicity, which suggests excellent potential for in vivo biomedical applications.

\section{Targeting efficiency in vitro}

To investigate the targeting ability of the SPIO/DSPEPEG5k-(Bom\&Cy5) nanomicelles, a cell uptake study using MDA-MB-231 cells was performed. The Bom-targeted nanomicelles were incubated with cells and compared to two controls: Bom-targeted nanomicelles with free Bom and nontargeted nanomicelles. After incubation for $4 \mathrm{hrs}$, the cellular uptake was observed by a fluorescence microscope. Strong Cy5 fluorescence revealed that a large number of nanomicelles were attached to and entered the MDA-MB-231 cells incubated with Bom-targeted nanomicelles, while weak fluorescence was observed from the nontargeted group (Figure 5). Blocking the receptor-binding sites with free Bom decreased the uptake of targeted nanomicelles to the level of the nontargeted nanomicelles (Figure 5), indicating that this imaging nanomicelle does in fact undergo internalization upon receptor binding. These observations demonstrate that the Bom-targeted nanomicelles exhibited a strong targeting ability toward GRPR-positive cells and might be utilized as a prospective active targeting contrast agent.

\section{In vivo targeting and imaging performance}

We then tested the dual-modality imaging properties of the nanomicelles in vivo using MDA-MB-231 breast xenograft models in nude mice. Once the tumors reached 5$10 \mathrm{~mm}$ in diameter, the nude mice were administered 200 $\mu \mathrm{L}(100 \mu \mathrm{g} \mathrm{Fe} / \mathrm{mL})$ of SPIO/DSPE-PEG5k-(Bom\&Cy5) or SPIO/DSPE-PEG5k-Cy5 nanomicelles via the tail vein. 


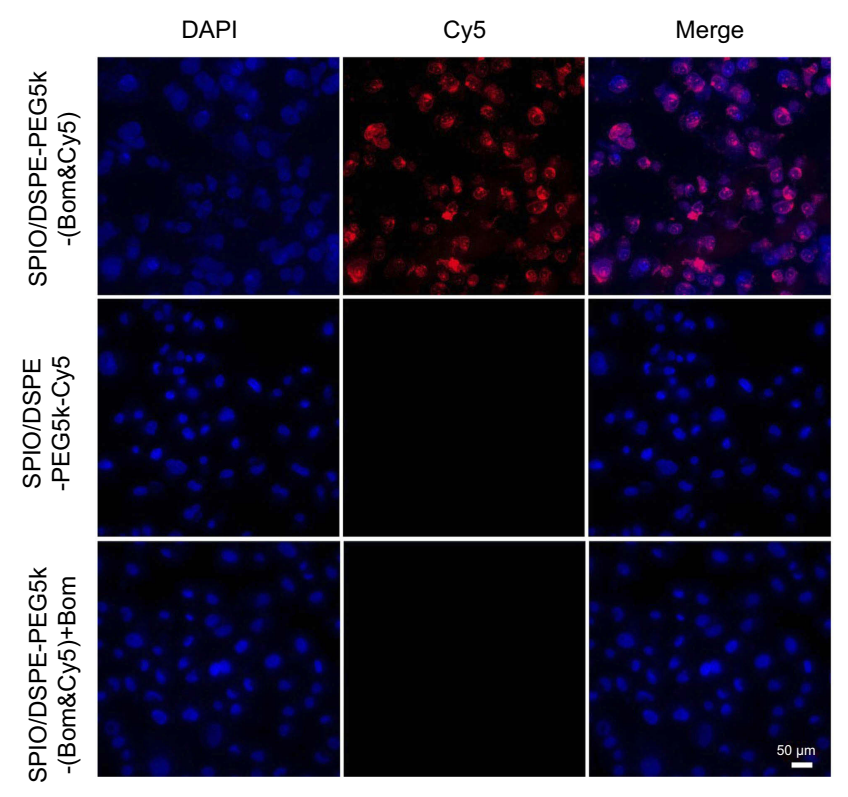

Figure 5 Targeting performance in cells.

Notes: Fluorescence microscopy of MDA-MB-23। cells incubated with Bom-targeted nanomicelles (Ist line), nontargeted nanomicelles (2nd line) and Bom-targeted nanomicelles with free Bom ( $3 r d$ line). From left to right are DAPI-labeled nuclei, Cy5-labeled nanomicelles and their overlay (scale bar $=50 \mu \mathrm{m}$ ).

All experiments were performed under complete anesthesia using $3 \%$ isoflurane.

In vivo $T_{2}$-weighted MRI was performed preinjection and 1, 2 and $4 \mathrm{hrs}$ after injection, respectively. The NSI was acquired by the $\mathrm{SI}_{\text {tumor }}$ divided by the $\mathrm{SI}_{\text {water phantom. For }}$ mice receiving the Bom-targeted nanomicelles, the tumor region in the $T_{2}$-weighted images became darker over time (Figure 6A), indicating decreased tumor MR SI after injection. For mice receiving the nontargeted nanomicelles, almost no changes in the signal from the tumor site were observed (Figure 6A). MRI scans performed before and 4 hrs after targeted nanomicelles injection showed a signal drop of $24.1 \pm 5.7 \%$ at the tumor sites, whereas only a 0.1 $\pm 3.4 \%(P=0.003)$ signal decrease was observed in the nontargeted group (Figure 6B). These results indicate that the SPIO/DSPE-PEG5k-(Bom\&Cy5) nanomicelles enhanced the contrast in tumor tissue and might be used as a $T_{2^{-}}$ negative contrast agent for MRI applications. NIRF images obtained at the same time points postinjection were then used to track in vivo delivery of the nanomicelles. For the targeted group, clear tumor delineation was observed at the $1 \mathrm{hr}$ time point, the contrast improved over time and the tumor/muscle ratio increased from $3.7 \pm 0.3$ at $1 \mathrm{hr}$ to 4.7 \pm 0.1 at 2 hrs and to $6.4 \pm 0.2$ at 4 hrs (Figure 6C and D). No significant changes were observed in the nontargeted group at any time points, showing a stable tumor/muscle ratio of
$1.6 \pm 0.1,1.8 \pm 0.1,1.9 \pm 0.2$ at $1 \mathrm{hr}, 2 \mathrm{hrs}$ and $4 \mathrm{hrs}$, respectively (Figure 6C and D). These results further confirm the targeting capacity of the Bom-targeted nanomicelles to GRPR-positive tumor tissue and indicate that the in vivo MR/NIRFI ability of the Bom-targeted nanomicelles was significantly superior to that of the nontargeted nanomicelles.

To improve our understanding of iron accumulation in tumor tissue, the tissue slices were stained with Prussian blue. Iron enrichment was detected in tumor cells from the Bom-targeted group (Figure 7A), while no significant accumulation was found in the nontargeted group (Figure 7B). This observation correlated well with the MR/NIRFI results.

\section{Discussion}

Multimodality imaging probes have emerged as powerful tools because of their potential for facilitating the early diagnosis of tumors with high accuracy. However, multimodality imaging probes face a challenge: they must integrate multiple imaging motifs with different levels of sensitivity. ${ }^{7,33}$ Biocompatibility and/or target efficiency should also be taken into account for probe design. Targeted nanoparticle-based design is likely the platform of choice for multimodality imaging probes owing to their easily controlled properties and good pharmacological characteristics. $^{34-37}$

In the present study, we developed a novel dual-modality MR/NIRFI nanomicelle by entrapping SPIO nanoparticles into DSPE-PEG5k micelles to which Cy5 and Bom were conjugated. Physical characterization showed that the near-neutral charged nanosized micelles were in the optimum range for enhancing cellular accumulation and biodistribution. ${ }^{38,39}$ The morphology of nanomicelles also plays a critical role in their cellular fate; spherical nanomicelles, as observed by TEM, show more efficient internalization by tumor cells. ${ }^{40,41}$

Considering that SPIO nanoparticles are FDA-approved drugs, we used them as biodegradable and biocompatible contrast agents for $T_{2}$-weighted MRI. A DSPE-PEG5k coating was used to improve the SPIO nanoparticles' solubility, circulation time and $r_{2} .{ }^{18,42}$ The obtained SPIO/DSPEPEG5k-(Bom\&Cy5) nanomicelles exhibited good biocompatibility and safety in mice (Figure 4), which is essential for their further clinical translation. The relaxivity characterization results show that the $r_{2}$ of the SPIO/DSPEPEG5k-(Bom\&Cy5) nanomicelles was $\sim 12$ times higher than that of free SPIO nanoparticles $\left(493.9 \mathrm{mM}^{-1} \cdot \mathrm{s}^{-1}\right.$ vs $\left.20-40 \mathrm{mM}^{-1} \cdot \mathrm{s}^{-1}\right),{ }^{43}$ mainly because the clustering of SPIO 

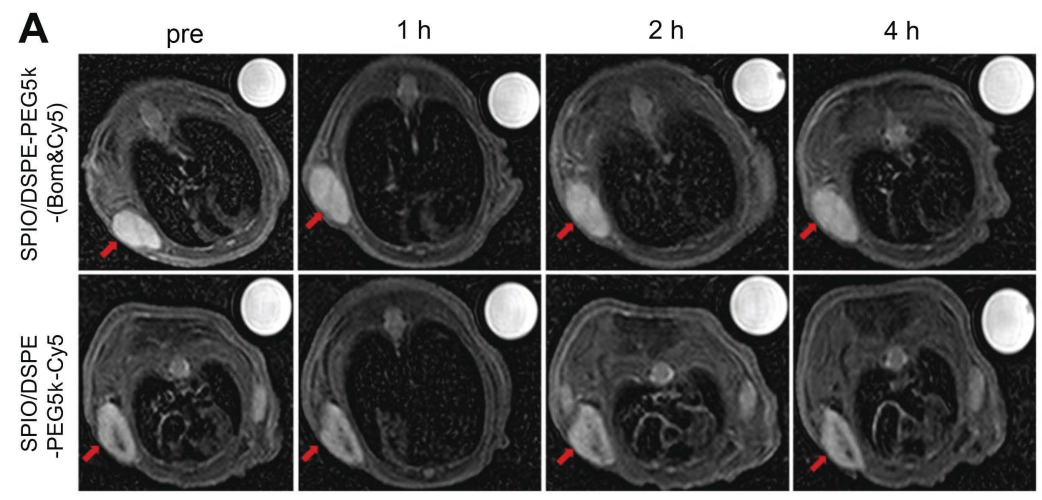

B

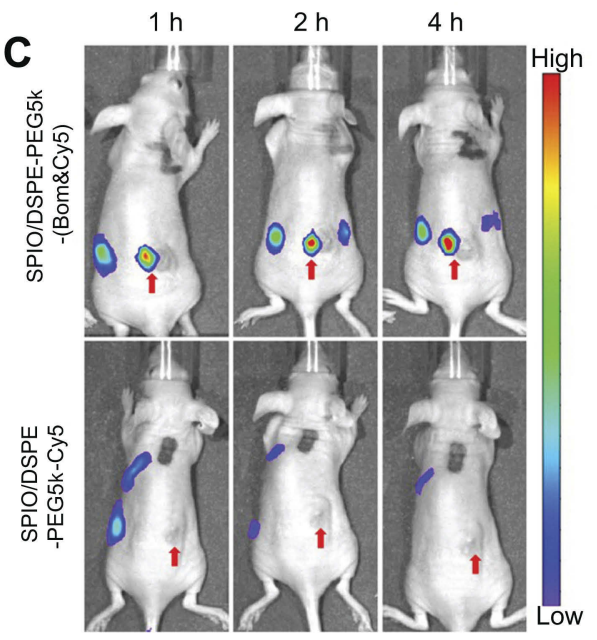

D

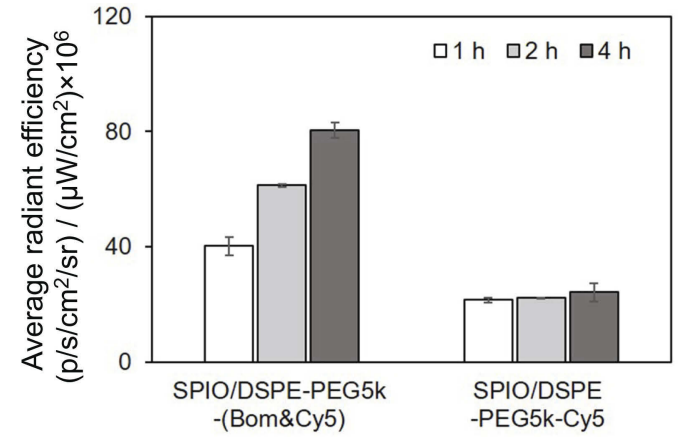

Figure 6 In vivo dual-modality imaging performance.

Notes: (A) MR images (3.0 T, $T_{2}$-weighted fast spin echo sequence: $T R=4000 \mathrm{~ms}$, TE $=66 \mathrm{~ms}, \mathrm{FOV}=50 \mathrm{~mm} \times 50 \mathrm{~mm}$, slice thickness $=\mathrm{I} \mathrm{mm}$ ) and (B) NSI of an MDA-MB-23। mouse xenograft tumor at different times after the intravenous injection of Bom-targeted nanomicelles and nontargeted nanomicelles. NSI=SI tumor $/ \mathrm{SI}_{\text {water phantom. }}$ (C) $\mathrm{NIRF}$ images and (D) average radiant efficiencies $\left(\mathrm{p} / \mathrm{s} / \mathrm{cm}^{2} / \mathrm{sr}\right) /\left(\mu \mathrm{W} / \mathrm{cm}^{2}\right)$ at different times after the injection of Bom-targeted or nontargeted nanomicelles. The red arrows indicate tumors. Abbreviations: NSI, normalized signal intensity; NIRF, near-infrared fluorescence.

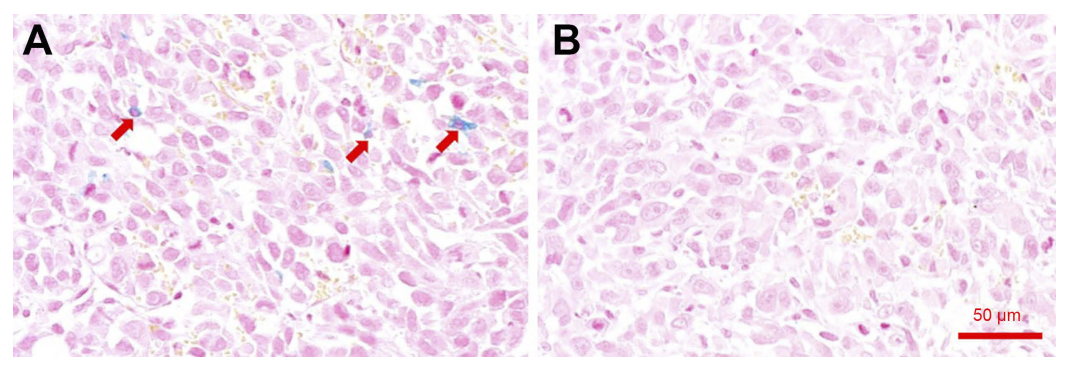

Figure 7 Prussian blue staining of tumor sections.

Notes: Prussian blue-stained tumor tissue section from mice treated with SPIO/DSPE-PEG5k-(Bom\&Cy5) nanomicelles (A) and SPIO/DSPE-PEG5k-Cy5 nanomicelles (B), respectively. The red arrows indicate SPIO nanoparticles. Scale bar $=50 \mu \mathrm{m}$.

Abbreviation: SPIO, superparamagnetic iron oxide.

nanoparticles inside micelle cores dramatically increases mass magnetization. ${ }^{42,44-46}$ A high relaxivity coefficient is a prerequisite for use as a novel $T_{2}$ negative contrast agent for sensitive MRI.

In addition, attaching a targeting group may facilitate the enrichment of SPIO nanoparticles at the desired sites and thereby enhance the sensitivity of MRI. Bom, a tumorhoming peptide, has many excellent properties, including small size, low immunogenicity, low cytotoxicity and easy synthesis. Radiolabeled Bom and its analogs have been reported to specifically target GRPR-overexpressing tumors. ${ }^{47-50}$ Therefore, it is expected that the tumor 
accumulation of SPIO nanoparticles in GRPR-overexpressing cells and tumors may be improved by conjugating Bom to the nanoparticle surface. Here, we used a facile click reaction to conjugate Bom to DSPE-PEG5k-Alky and confirmed its targeting capability in vitro and in vivo. Bom can bind specifically with GRPR overexpressed by MDA-MB231 cells (Figure 5), so we speculated that the MR signal drop and fluorescence signal in the nontargeted group were from the enhanced permeability and retention (EPR) effect, whereas the MR signal drop and fluorescence signal at the tumor site in the Bom-targeted group were mainly from the Bom-mediated active targeting effect. The active targeting effect was significantly stronger than the EPR effect of passive targeting at the tumor site.

The SPIO/DSPE-PEG5k-(Bom\&Cy5) nanomicelles exhibit high $r_{2}$, good biocompatibility and targeting specificity, and excellent image contrast in both MRI and NIRFI, which are essential requirements for imaging contrast agents in particular and for any further biomedical application in general. This research suggests that with the homing peptide Bom as a targeting mediator and DSPEPEG5k micelles as a carrier, the SPIO/DSPE-PEG5k(Bom\&Cy5) nanomicelles have promise for systematic toxicity reduction and tumor-targeted molecular imaging by MR and NIRF.

\section{Conclusion}

In this study, we successfully prepared and tested a dual-modality MR/NIRFI nanomicelle, SPIO/DSPEPEG5k-(Bom\&Cy5). We have demonstrated that these nanomicelles have a suitable size, high relaxivity and the ability to bind to the GRPR overexpressed on MDAMB-231 cells. Dual-modality imaging in vivo revealed that the SPIO/DSPE-PEG5k-(Bom\&Cy5) nanomicelles were effectively transported to the tumor tissue by a Bom-mediated active targeting effect. These findings suggest that the SPIO/DSPE-PEG5k-(Bom\&Cy5) nanomicelles, as a dual probe applicable for both MRI and NIRFI, are promising agents for the diagnosis of cancer.

\section{Acknowledgments}

This work was funded by the National Natural Science Foundation of China (nos. 81471693 and 81301250), the Key Projects of the Ministry of Science and Technology (no. 2017YFC0113304), and International Cooperative Project of Sichuan Provincial Science and Technology Department (no. 2016HH0065).

\section{Disclosure}

The authors report no conflicts of interest in this work.

\section{References}

1. Chen W, Zheng R, Baade PD, et al. Cancer statistics in China, 2015. CA Cancer J Clin. 2016;66(2):115-132. doi:10.3322/caac.21338

2. Stewart BW, Wild CP. World Cancer Report 2014. Lyon, France: International Agency for Research on Cancer; 2014.

3. Zheng XC, Ren W, Zhang S, et al. The theranostic efficiency of tumor-specific, $\mathrm{pH}$-responsive, peptide-modified, liposome-containing paclitaxel and superparamagnetic iron oxide nanoparticles. Int $J$ Nanomedicine. 2018;13:1495-1504. doi:10.2147/IJN.S157082

4. Kim JS, Kim YH, Kim JH, et al. Development and in vivo imaging of a PET/MRI nanoprobe with enhanced NIR fluorescence by dye encapsulation. Nanomedicine (Lond). 2012;7(2):219-229. doi: $10.2217 / \mathrm{nnm} .11 .94$

5. Bennett KM, Jo J, Cabral H, Bakalova R, Aoki I. MR imaging techniques for nano-pathophysiology and theranostics. Adv Drug Deliv Rev. 2014;74:75-94. doi:10.1016/j.addr.2014.04.007

6. Albanese C, Rodriguez OC, VanMeter J, et al. Preclinical magnetic resonance imaging and systems biology in cancer research: current applications and challenges. Am J Pathol. 2013;182(2):312-318. doi:10.1016/j.ajpath.2012.09.024

7. Zhao J, Chen J, Ma S, et al. Recent developments in multimodality fluorescence imaging probes. Acta Pharm Sin B. 2018;8(3):320-338. doi:10.1016/j.apsb.2018.03.010

8. Haque A, Faizi M, Rather JA, Khan MS. Next generation NIR fluorophores for tumor imaging and fluorescence-guided surgery: a review. Bioorg Med Chem. 2017;25(7):2017-2034. doi:10.1016/j. bmc.2017.02.061

9. Yi X, Wang F, Qin W, Yang X, Yuan J. Near-infrared fluorescent probes in cancer imaging and therapy: an emerging field. Int $J$ Nanomedicine. 2014;9:1347-1365. doi:10.2147/IJN.S60206

10. Vallabani N, Singh S. Recent advances and future prospects of iron oxide nanoparticles in biomedicine and diagnostics. 3 Biotech. 2018;8(6):279. doi:10.1007/s13205-018-1286-z

11. Blanco-Andujar C, Walter A, Cotin G, et al. Design of iron oxidebased nanoparticles for MRI and magnetic hyperthermia. Nanomedicine (Lond). 2016;11(14):1889-1910. doi:10.2217/nnm2016-5001

12. Deng L, Cai X, Sheng D, et al. A laser-activated biocompatible theranostic nanoagent for targeted multimodal imaging and photothermal therapy. Theranostics. 2017;7(18):4410-4423. doi:10.7150/ thno. 21283

13. Chen Q, Shang W, Zeng C, et al. Theranostic imaging of liver cancer using targeted optical/MRI dual-modal probes. Oncotarget. 2017;8 (20):32741-32751. doi:10.18632/oncotarget.15642

14. Kim D, Rozhkova EA, Rajh T, Bader SD, Novosad V. Synthesis of hybrid gold/iron oxide nanoparticles in block copolymer micelles for imaging, drug delivery, and magnetic hyperthermia. Ieee $T$ Magn. 2009;45(10):4821-4824. doi:10.1109/TMAG.2 009.2024000

15. Vitol EA, Rozhkova EA, Rose V, et al. Efficient cisplatin pro-drug delivery visualized with sub- $100 \mathrm{~nm}$ resolution: interfacing engineered thermosensitive magnetomicelles with a living system. $A d v$ Mater Interfaces. 2014;1(7):1400182. doi:10.1002/admi.2014 00182

16. Veiseh O, Gunn JW, Zhang M. Design and fabrication of magnetic nanoparticles for targeted drug delivery and imaging. Adv Drug Deliv Rev. 2010;62(3):284-304. doi:10.1016/j.addr.2009.11.002

17. Ai H, Flask C, Weinberg B, et al. Magnetite-loaded polymeric micelles as ultrasensitive magnetic-resonance probes. Adv Mater. 2005;17(16):1949-1952. doi:10.1002/adma.200401904 
18. Upponi JR, Jerajani K, Nagesha DK, et al. Polymeric micelles: theranostic co-delivery system for poorly water-soluble drugs and contrast agents. Biomaterials. 2018;170:26-36. doi:10.1016/j. biomaterials.2018.03.054

19. Wang R, Xiao R, Zeng Z, Xu L, Wang J. Application of poly(ethylene glycol)-distearoylphosphatidylethanolamine (PEG-DSPE) block copolymers and their derivatives as nanomaterials in drug delivery. Int $J$ Nanomedicine. 2012;7:4185-4198. doi:10.2147/IJN.S34489

20. Xie W, Guo Z, Gao F, et al. Shape-, size- and structure-controlled synthesis and biocompatibility of iron oxide nanoparticles for magnetic theranostics. Theranostics. 2018;8(12):3284-3307. doi:10.7150/ thno. 25220

21. Che J, Okeke CI, Hu ZB, Xu J. DSPE-PEG: a distinctive component in drug delivery system. Curr Pharm Des. 2015;21(12):1598-1605.

22. Muthiah M, Park I, Cho C. Surface modification of iron oxide nanoparticles by biocompatible polymers for tissue imaging and targeting. Biotechnol Adv. 2013;31(8):1224-1236. doi:10.1016/j. biotechadv.2013.03.005

23. Ferreira CDA, Fuscaldi LL, Townsend DM, Rubello D, Barros ALBD. Radiolabeled bombesin derivatives for preclinical oncological imaging. Biomed Pharmacother. 2017;87:58-72. doi:10.1016/j. biopha.2016.12.083

24. Moreno P, Ramos-Álvarez I, Moody TW, Jensen RT. Bombesin related peptides/receptors and their promising therapeutic roles in cancer imaging, targeting and treatment. Expert Opin Ther Tar. 2016;20(9):1055-1073. doi:10.1517/14728222.2016.1164694

25. Sancho V, Di Florio A, Moody TW, Jensen RT. Bombesin receptormediated imaging and cytotoxicity: review and current status. Curr Drug Deliv. 2011;8(1):79-134.

26. Ramos-Álvarez I, Moreno P, Mantey SA, et al. Insights into bombesin receptors and ligands: highlighting recent advances. Peptides. 2015;72:128-144. doi:10.1016/j.peptides.2015.04.026

27. Jafari A, Salouti M, Shayesteh SF, et al. Synthesis and characterization of Bombesin-superparamagnetic iron oxide nanoparticles as a targeted contrast agent for imaging of breast cancer using MRI. Nanotechnology. 2015;26(7):75101. doi:10.1088/0957-4484/26/7/ 075101

28. Martin AL, Hickey JL, Ablack AL, Lewis JD, Luyt LG, Gillies ER. Synthesis of bombesin-functionalized iron oxide nanoparticles and their specific uptake in prostate cancer cells. J Nanopart Res. 2009;12 (5):1599-1608. doi:10.1007/s11051-009-9681-3

29. Lee CM, Jeong HJ, Cheong SJ, et al. Prostate cancer-targeted imaging using magnetofluorescent polymeric nanoparticles functionalized with bombesin. Pharm Res. 2010;27(4):712-721. doi:10.1007/ s11095-010-0072-3

30. Montet X, Weissleder R, Josephson L. Imaging pancreatic cancer with a peptide-nanoparticle conjugate targeted to normal pancreas. Bioconjug Chem. 2006;17(4):905-911. doi:10.1021/bc060035+

31. Sun $\mathrm{S}$, Zeng $\mathrm{H}$, Robinson $\mathrm{DB}$, et al. Monodisperse $\mathrm{MFe}_{2} \mathrm{O}_{4}(\mathrm{M}=\mathrm{Fe}$, Co, Mn) nanoparticles. J Am Chem Soc. 2004;126(1):273-279. doi:10.1021/ja0380852

32. Inoue Y, Izawa K, Yoshikawa K, Yamada H, Tojo A, Ohtomo K. In vivo fluorescence imaging of the reticuloendothelial system using quantum dots in combination with bioluminescent tumour monitoring. Eur J Nucl Med Mol Imaging. 2007;34(12):2048-2056. doi:10.1007/s00259-007-0583-2

33. Louie A. Multimodality imaging probes: design and challenges. Chem Rev. 2010;110(5):3146-3195. doi:10.1021/cr9003538
34. Ehlerding EB, Grodzinski P, Cai W, Liu CH. Big potential from small agents: nanoparticles for imaging-based companion diagnostics. $A C S$ Nano. 2018;12(3):2106-2121. doi:10.1021/acsnano.7b07252

35. Xing Y, Zhao J, Conti PS, Chen K. Radiolabeled nanoparticles for multimodality tumor imaging. Theranostics. 2014;4(3):290-306. doi: $10.7150 /$ thno.7341

36. Hsu JC, Naha PC, Lau KC, et al. An all-in-one nanoparticle (AION) contrast agent for breast cancer screening with DEM-CT-MRI-NIRF imaging. Nanoscale. 2018;10(36):17236-17248. doi:10.1039/ c8nr03741h

37. Xie J, Chen K, Huang J, et al. PET/NIRF/MRI triple functional iron oxide nanoparticles. Biomaterials. 2010;31(11):3016-3022. doi:10.1016/j.biomaterials.2010.01.010

38. Kim E, Kim JM, Kim L, et al. The effect of neutral-surface iron oxide nanoparticles on cellular uptake and signaling pathways. Int $J$ Nanomedicine. 2016;11:4595-4607. doi:10.2147/IJN.S110332

39. Sadat SMA, Jahan ST, Haddadi A. Effects of size and surface charge of polymeric nanoparticles on in vitro and in vivo applications. $J$ Biomater Nanobiotechnol. 2016;07(02):91-108. doi:10.4236/ jbnb.2016.72011

40. Qiu Y, Liu Y, Wang L, et al. Surface chemistry and aspect ratio mediated cellular uptake of Au nanorods. Biomaterials. 2010;31 (30):7606-7619. doi:10.1016/j.biomaterials.2010.06.051

41. Bartneck M, Keul HA, Singh S, et al. Rapid uptake of gold nanorods by primary human blood phagocytes and immunomodulatory effects of surface chemistry. ACS Nano. 2010;4(6):3073-3086. doi:10.1021/ $\mathrm{nn} 100262 \mathrm{~h}$

42. Zhang W, Liu L, Chen H, et al. Surface impact on nanoparticle-based magnetic resonance imaging contrast agents. Theranostics. 2018;8 (9):2521-2548. doi:10.7150/thno.23789

43. Zhu L, Wang D, Wei X, et al. Multifunctional pH-sensitive superparamagnetic iron-oxide nanocomposites for targeted drug delivery and MR imaging. J Control Release. 2013;169(3):228-238. doi:10.1016/j.jconrel.2013.02.015

44. Nasongkla N, Bey E, Ren J, et al. Multifunctional polymeric micelles as cancer-targeted, MRI-ultrasensitive drug delivery systems. Nano Lett. 2006;6(11):2427-2430. doi:10.1021/n1061412u

45. Tong S, Hou S, Zheng Z, Zhou J, Bao G. Coating optimization of superparamagnetic iron oxide nanoparticles for high $\mathrm{T}_{2}$ relaxivity. Nano Lett. 2010;10(11):4607-4613. doi:10.1021/nl102623x

46. Kim DH, Vitol EA, Liu J, et al. Stimuli-responsive magnetic nanomicelles as multifunctional heat and cargo delivery vehicles. Langmuir. 2013;29(24):7425-7432. doi:10.1021/la3044158

47. Stoykow C, Erbes T, Maecke HR, et al. Gastrin-releasing peptide receptor imaging in breast cancer using the receptor antagonist ${ }^{68} \mathrm{Ga}$ RM2 and PET. Theranostics. 2016;6(10):1641-1650. doi:10.7150/ thno. 14958

48. Zang J, Mao F, Wang H, et al. ${ }^{68}$ Ga-NOTA-RM26 PET/CT in the evaluation of breast cancer: a pilot prospective study. Clin Nucl Med. 2018;43(9):663-669. doi:10.1097/RLU.0000000000002209

49. Cai H, Xie F, Mulgaonkar A, et al. Bombesin functionalized ${ }^{64} \mathrm{Cu}-$ copper sulfide nanoparticles for targeted imaging of orthotopic prostate cancer. Nanomedicine (Lond). 2018;13. doi:10.2217/nnm2018-0062

50. Zhou Z, Wagh NK, Ogbomo SM, et al. Synthesis and in vitro and in vivo evaluation of hypoxia-enhanced ${ }^{111} \mathrm{In}$-bombesin conjugates for prostate cancer imaging. J Nucl Med. 2013;54(9):1605-1612. doi:10.2967/jnumed.112.117986 


\section{Publish your work in this journal}

The International Journal of Nanomedicine is an international, peerreviewed journal focusing on the application of nanotechnology in diagnostics, therapeutics, and drug delivery systems throughout the biomedical field. This journal is indexed on PubMed Central, MedLine, CAS, SciSearch ${ }^{\circledR}$, Current Contents ${ }^{\circledR} /$ Clinical Medicine,
Journal Citation Reports/Science Edition, EMBase, Scopus and the Elsevier Bibliographic databases. The manuscript management system is completely online and includes a very quick and fair peer-review system, which is all easy to use. Visit http://www.dovepress.com/ testimonials.php to read real quotes from published authors. 